\title{
EL "PROBLEMA DE LA DROGA" Y LA PREVENCIÓN DEL CONSUMO DE DROGAS: MITOS Y ALTERNATIVAS, CON UNA ESPECIAL REFERENCIA AL CONSUMO DE CANNABIS
}

\author{
SALVADOR AMIGÓ BORRÁS
}

Profesor Asociado de la Facultad de Psicología de la Universidad de Valencia.

\section{RESUMEN}

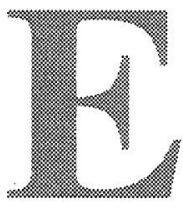

n este estudio se realiza una investigación básica sobre los aspectos que fundamentan los programas de prevención del consumo de drogas y la actual política de drogas, que tiene como objetivo afrontar el "problema de la droga". Así, se estudian los determinantes del consumo, dependencia y abuso de cannabis, así como la aceptación de mensajes y consejos sobre su consumo (permisivos versus punitivos). Se cuenta con una muestra de 70 jóvenes, de los que 33 son consumidores frecuentes de cannabis, 16 son no consumidores, 10 consumidores experimentales y el resto consumidores de otras drogas. Se les administró una amplia encuesta compuesta de dos partes: una batería de pruebas psicológicas (EPQ, inventario de calidad de vida, listado de problemas, inventario de felicidad, cuestionario de modos de afrontamiento y cuestionario de emocionalidad) y un cuestionario de drogas (incidencia del consumo de drogas, patrones de consumo, criterios de dependencia y abuso, efectos a corto y largo plazo, actitudes y motivaciones, y preferencia por mensajes permisivos o punitivos del consumo). Los resultados indican que hay una diferencia significativa entre los consumidores frecuentes y los no consumidores en variables estables como psicoticismo e inconformismo. Por otra parte, los consumidores frecuentes aceptan mucho más los mensajes permisivos sobre el consumo y los consejos para prevenir consecuencias adversas (reducción de daño) que los no consumidores, que prefieren los mensajes punitivos. Además, los consumidores frecuentes de cannabis informan de unos niveles importantes de control del consumo, y unos beneficios percibidos del consumo prolongado en mayor medida que las consecuencias negativas. Estos resultados serán interpretados en el contexto del fracaso de los programas de prevención del consumo de 
drogas y de la actual política prohibicionista de lucha contra la droga, proponiendo alternativas en ambos aspectos.

\section{INTRODUCCIÓN}

Una de las prioridades de los gobiernos occidentales en materia política y sanitaria es la lucha contra la droga. Las medidas que se implementan para ello son tanto legislativas y policiales como preventivas y asistenciales. Es el abordaje preventivo de la drogadicción lo que nos servirá como punto de partida de este estudio.

En la actualidad, proliferan gran cantidad de programas de prevención, aplicados en los ámbitos comunitario y escolar, y que gozan de una gran aceptación en la opinión pública. Normalmente, se entiende, por una parte, que los gobiernos deben promover estos programas, ya que una de sus funciones es afrontar el problema de la droga $y$, por otra parte, que los programas de prevención son eficaces en la consecución de su propósito de reducir el consumo de droga. Ahora bien, esto nos lleva a lo que considero los dos mitos asociados a los programas de prevención del consumo de drogas: la eficacia de los mismos y que exista el problema de las drogas. Digamos algo primero sobre la eficacia de los programas de prevención.

En un principio, hay que señalar que una grandísima proporción de los programas de prevención no presentan los requisitos mínimos para poder ser evaluados, a pesar del énfasis que sus promotores ponen en los aspectos metodológicos (sic) en la implementación de tales programas. De una revisión de 350 programas recogidos para su análisis en los años 1989 y 1990 en España, sólo 100 reunían condiciones mínimas para ser incluidos en las bases de datos, y de estos, sólo el $10 \%$ presentaba resultados relacionados con las hipótesis de la aplicación del programa, y el 5\% hacía una valoración de dichos resultados de acuerdo con sus objetivos (Froján y Santacreu, 1994). En algunos estudios sobre la eficacia de los programas de prevención (Goodstadt, 1978; Goodstadt, Sheppard y Chan, 1982) es difícil extraer resultados concluyentes al contar con errores metodológicos, como el no distinguir los diferentes tipos de programas (específicos vs. inespecíficos, información-cambio de actitud, etc.) que se están comparando (Smart, 1983). Así, parece ser que los programas basados fundamentalmente en la información sobre las drogas y sus consecuencias no son efectivos en modificar las actitudes hacia la droga $\mathrm{y}$, cuando lo consiguen, no siempre es en la dirección esperada (Pickens, 1985). Por ptra parte, los programas educativo-formativos, donde se utilizan fundamentalmente estrategias de información, persuasión y 
otras actividades escolares, sí consiguen un cambio significativo en las creencias y actitudes hacia la droga (Bangert-Drowns, 1988; Escámez, 1990). No obstante, no está claro si estos programas consiguen el cambio no sólo de actitud sino de conducta futura de los jóvenes respecto a la droga (para una discusión del cambio de actitudes y del comportamiento sobre la droga, ver Maciá, 1986). Esto ha hecho que muchos programas incorporen entrenamiento, con modelado y role-playing, para resistir la presión de los pares para consumir, consiguiéndose resultados satisfactorios en la reducción del consumo del tabaco (Biglan, Weissman y Severson, (1985), así como del tabaco y el alcohol desde la perspectiva de la competencia social y la autoestima (Pentz, 1985).

Uno de los programas más importantes en el mundo, diseñado para resistirse a la presión de los pares, aumentar la autoestima y la aceptación de las instituciones, así como la eliminación del consumo de sustancias y el retraso en la edad de la experimentación con alcohol, tabaco y otras drogas, es el DARE (Drug Abuse Resistance Education). Está diseñado para los preadolescentes, poniendo énfasis en los mensajes anti-droga y en las destrezas de resistencia a la presión, antes de acceder a la escuela media. Durante un semestre y una vez a la semana, un policía entrenado para tal propósito, desarrolla el contenido del programa. No hay duda de que el DARE es el programa de prevención del consumo de drogas más difundido en los Estados Unidos (Ennet et al., 1994). De ser un programa de ámbito local en 1983 en Los Angeles ha pasado a ser un proyecto internacional representado en más de 20 países (Becker, Agopian y Yeh, 1992). Es, además, un programa muy bien aceptado y valorado por los preadolescentes y los adultos (Wysong, Anikiewick y Wright, 1994). Por tratarse de un programa tan importante y difundido y por estar constituido por aspectos que se consideran básicos en este tipo de programas (mensajes antidroga, mejora de la autoestima, habilidades para resistirse a la influencia de los amigos, etc.), nos parece especialmente relevante detenernos por un momento en los estudios que desde sus comienzos se han realizado para comprobar la eficacia del mismo.

Una revisión reciente de los programas DARE (Dukes, Ullman y Stein, 1996) concluye que, mientras estos programas han demostrado su eficacia a corto plazo, en el largo plazo (un año o más) no se muestran efectivos. Así, mientras estos autores obtuvieron en 1995 resultados significativos del DARE en un estudio longitudinal de 6 meses, no obtuvieron diferencias en autoestima, resistencia a la presión de los pares y consumo en un estudio posterior de 3 años de seguimiento (Dukes, Ullman y Stein, 1996). Así, estudios como el de Ennett, Rosembaum et al. (1994) encontraron unos efectos del DARE en el 
corto plazo en medidas de conocimiento de drogas, autoestima, habilidades sociales, actitudes hacia la policía y hacia el consumo de droga, así como al propio consumo de droga en el grupo que recibió el programa frente al grupo de control. Cuando se evaluó entre 1 y 2 años después de terminado el programa, no se obtuvieron diferencias significativas ni en el consumo de tabaco ni en el de alcohol. Resultados similares fueron obtenidos por Faine y Bohlander (1989). Estudios como el de Clayton et al. (1991) encontraron que inmediatamente después del DARE, los participantes tenían una actitud anti-droga claramente marcada, pero, un año después (Clayton, Cattarello y Walden, 1991), tan sólo encontraron una diferencia entre el grupo experimental y el de control, y es que los jóvenes que recibieron el programa consumían más marihuana que los controles. Algunos efectos paradójicos más se han obtenido con la implementación del DARE, encontrándose un mayor consumo de alucinógenos en aquellos que participaron en el programa frente a los controles (Wyson, Aniskiewick y Wright, 1994). Autores como Dukes, Ullman y Stein (1996) y Rosembaum et al. (1994) indican que este tipo de resultados ponen de manifiesto la necesidad de llevar a cabo programas más prolongados en el tiempo donde los temas sean tratados con más profundidad. Ahora bien, no sabemos con certeza si esta modificación en la aplicación del programa, independientemente del problema de un mayor coste económico, ofrecerá mejores resultados a largo plazo. Así, el DARE va dirigido a los preadolescentes, de manera que, con el paso del tiempo, la experimentación y el consumo de drogas aumentará en esta población, lo que significa que los efectos madurativos van en dirección opuesta al pretendido efecto de estos programas (Dukes, Ullman y Stein, 1995). Además, con la edad, los adolescentes se hacen más conscientes de la distinción entre uso y abuso de drogas y se vuelven mucho más escépticos a los mensajes anti-droga de estos programas, a los que empiezan a dar una valoración más negativa (Brown y Caston, 1995).

Podemos concluir que, aunque está aceptada socialmente la implementación de programas de prevención del consumo de drogas, y aunque las autoridades sanitarias y los profesionales elogien sus beneficios, el rigor metodológico y la eficacia de estos programas está muy lejos de lo que éstos preconizan. Incluso se ha constatado, como anteriormente hemos señalado, que algunos de estos programas pueden tener efectos contrapreventivos. Así se ha construido el mito de la necesidad y eficacia de los programas de prevención del consumo de drogas sobre un soporte más que inconsistente. No es de extrañar que en este estado de cosas se considere innecesaria la investigación básica y la incorporación de los resultados de investigación en los progra- 
mas de prevención (Dembo, 1979, 1981), lo que a su vez puede explicar el escaso éxito de estos programas.

Precisamente, este es el objetivo fundamental de este estudio: realizar investigación básica que ahonde en los determinantes del consumo y abuso, así como en la preferencia por diferentes mensajes y consejos referidos a las drogas. Espero que este estudio de carácter exploratorio aporte algunas cuestiones concretas que nos alerten sobre las razones del aparente fracaso de los programas de prevención, así como de las posibles soluciones a lo que he llamado problema de la droga y que más adelante será tratado con algún detalle. Si bien este estudio recoge información relevante para varias drogas (cannabis, éxtasis y cocaína), en este artículo sólo se presentarán los resultados de las drogas en general y del cannabis en particular, dejando los resultados referentes al éxtasis y a la cocaína para un futuro escrutinio.

\section{SUJETOS, MATERIALES, PROCEDIMIENTO Y MÉTODO}

De un total de 80 encuestas sobre la droga, se eliminaron 10 casos por errores importantes o datos perdidos, quedando la muestra constituida por 70 personas. La muestra se obtuvo a partir de la colaboración de un grupo de estudiantes de primer año de universidad a los que se encuestó, los cuales a su vez localizaron a personas que consumían o bien cannabis (hachis/marihuana) o bien éxtasis y/o cocaína, para administrarles las pruebas. Así, no se trata de ningún estudio sobre incidencia en el consumo de drogas en una muestra determinada, sino un estudio sobre los determinantes del consumo en una muestra heterogénea en la que hay una mayor proporción de consumidores de la que se obtendría en la población general.

La edad media de estas personas es de 19 años, con una desviación típica de 3,16 y con un rango de 16-36. De ellos, $30(42,9 \%)$ eran hombres y $40(57,1 \%)$ mujeres. La mayoría eran estudiantes de primer ciclo de universidad $(71,4 \%)$ y de BUP y COU $(15,7 \%)$ siendo el resto estudiantes de FP, trabajadores no cualificados y desempleados.

La encuesta está compuesta por dos partes: batería de pruebas psicológicas y cuestionario sobre drogas.

- Batería de pruebas psicológicas:

- Cuestionario de Personalidad de Eysenck (EPQ-Eysenck y Eysenck, 1975).

- Inventario de Calidad de Vida (ICV-R, García Riaño, en preparación).

- Inventario de Felicidad de Oxford (Argyle y Lu, 1990).

- Listado de Problemas (McKay, Davis y Fanning, 1981). 
- Cuestionario de Modos de Afrontamiento (Holahan y Moosm 1987).

- Cuestionario de Creencias de Control Emocional (NMRCataranzo y Mears, 1990).

- Escala de Afecto Positivo y Negativo (PANAS- Watson, Clark y Tellegen, 1988).

- Cuestionario sobre drogas.

Construido para esta investigación. Consta de varios apartados:

1. Tabla de incidencias de consumo de varias drogas, tanto en el pasado como en el presente, y consumo en el último mes.

2. Patrones de consumo de cannabis, éxtasis y cocaína.

3. Preguntas basadas en los criterios del DSM-IV para el diagnóstico de dependencia y abuso de drogas.

4. Listados de efectos a corto y largo plazo del cannabis, éxtasis y cocaína, así como las motivaciones para el consumo de cada una de ellas y el nivel percibido de control.

5. Listado de actitudes hacia las drogas en general.

6. Mensajes informativos y persuasivos sobre el consumo de drogas. Para cada una de las tres drogas (cannabis, éxtasis y cocaína), se presentan aleatorizados dos mensajes alternativos: uno de ellos da un mensaje negativo sobre la droga e intenta persuadir de su no consumo. El otro mensaje es más permisivo sobre el consumo, dando consejos para evitar consecuencias negativas del mismo. Se trata así de presentar un mensaje punitivo y otro permisivo y de reducción de daño sobre las drogas. A los sujetos se les decía que ambos mensajes son correctos y que ninguno de ellos pretende engañarle. Tras la lectura de los dos mensajes, cada sujeto respondía a las mismas 6 preguntas para cada droga y que son :

- ¿Cuál de los dos mensajes te parece más interesante?

- ¿Cuál estarías, de verdad, dispuesto a seguir?

- ¿Cuál te parece más beneficioso para ti?

- ¿Cuál te crees más?

- ¿Con cuál te identificas más?

- ¿Cuál darías antes a un amigo que consume la droga?

Una vez cumplimentados los cuestionarios, se procedió a realizar una gran cantidad de análisis estadísticos, de los cuales se extraerán únicamente los más relevantes para ser presentados aquí. 


\section{III.RESULTADOS}

En primer lugar, vamos a considerar la incidencia del consumo de drogas en nuestra muestra, tanto en el presente como en el pasado. Hay que resaltar que estas dos categorías son excluyentes. Así, consideramos ANTES como el consumo de una determinada sustancia en el pasado, pero que se interrumpió en algún momento y no persiste en el presente, mientras que en la categoría AHORA incluimos los consumos actuales independientemente de que fueran interrumpidos en el pasado. En el CUADRO 1 se presentan estos datos.

CUADRO 1: Incidencia del consumo de drogas en la muestra de este estudio.

\begin{tabular}{|lrlrl|}
\hline & ANTES & \multicolumn{3}{c|}{ AHORA } \\
Cannabis & 21 & $(30 \%)$ & 30 & $(42,9 \%)$ \\
Extasis & 12 & $(17,1 \%)$ & 7 & $(10 \%)$ \\
Cocaína & 18 & $(25,7 \%)$ & 8 & $(11,4 \%)$ \\
Tabaco & 18 & $(25,7 \%)$ & 39 & $(55,7 \%)$ \\
Alcohol & 14 & $(20 \%)$ & 51 & $(72,9 \%)$ \\
Anfetaminas & 8 & $(11,4 \%)$ & 1 & $(1,4 \%)$ \\
Heroína & 1 & $(1,4 \%)$ & 0 & $(0 \%)$ \\
Opiáceos & 6 & $(8,6 \%)$ & 3 & $(4,3 \%)$ \\
Rohipnol & 1 & $(1,4 \%)$ & 0 & $(0 \%)$ \\
Inhalantes & 8 & $(11,4 \%)$ & 1 & $(1,4 \%)$ \\
Alucinógenos & 18 & $(25,7 \%)$ & 3 & $(4,3 \%)$ \\
Tranquilizantes & 9 & $(12,9 \%)$ & 3 & $(4,3 \%)$ \\
\hline
\end{tabular}

EI CUADRO 2 recoge las actitudes sobre las drogas y las respuestas a las preguntas anteriormente formuladas sobre la preferencia de los mensajes sobre drogas, a los que llamaremos PERMISIVO $\mathrm{Y}$ PUNITIVO.

CUADRO 2: Actitudes ante la droga y los mensajes para la muestra total (en \%).

\begin{tabular}{|lr|}
\hline La droga es mala & 42,9 \\
La droga es buena & 1,4 \\
La droga no es buena ni mala & 2,9 \\
Depende de la droga & 14,3 \\
Depende de cómo se utilice & 37,1 \\
(En las siguientes preguntas se permitía más de una respuesta) & \\
Ayuda a mejorar las relaciones & 7,1 \\
Es muy peligrosa para la salud & 64,3 \\
Debería penalizarse su consumo & 12,9 \\
Es difícil controlar su consumo & 41,4 \\
Debería estar legalizada & 37,1 \\
Quien consume es que no sabe divertirse sin droga & 31,4 \\
Hace que te lo pases mejor con los amigos & 15,7 \\
\hline
\end{tabular}




\begin{tabular}{|lcc|}
\hline MENSAJES CANNABIS & PUNITIVO & PERMISIVO \\
1. Interesante & 44,3 & 54,3 \\
2. Dispuesto a seguir & 50 & 48,6 \\
3. Beneficioso & 58,6 & 40 \\
4. Más creíble & 50 & 48,6 \\
5. Te identificas & 45,7 & 52,9 \\
6. Dar a un amigo que consume & 52,9 & 45,7 \\
MENSAJES EXTASIS & PUNITIVO & PERMISIVO \\
1. Interesante & 52,9 & 47,1 \\
2. Dispuesto a seguir & 65,7 & 34,3 \\
3. Beneficioso & 84,3 & 15,7 \\
4. Más creíble & 70 & 30 \\
5. Te identificas & 64,3 & 35,7 \\
6. Dar a un amigo que consume & 65,7 & 34,3 \\
MENSAJES COCAÍNA & PUNITIVO & PERMISIVO \\
1. Interesante & 54,3 & 45,7 \\
2. Dispuesto a seguir & 74,3 & 25,7 \\
3. Beneficioso & 80 & 20 \\
4. Más creíble & $7 I, 4$ & 28,6 \\
5. Te identificas & 70 & 30 \\
6. Dar a un amigo que consume & 71,4 & 28,6 \\
\hline
\end{tabular}

De estos resultados, podemos destacar que para la mitad de los jóvenes de esta muestra la droga es claramente mala, y que para un tercio esto depende de la droga o de cómo se utilice. Más de la mitad cree que las drogas son muy peligrosas para la salud y que es muy difícil controlarlas. En cuanto a los mensajes, lo primero destacable es la mayor aceptación del cannabis frente al éxtasis y la cocaína. Así, mientras que para el cannabis se reparte a partes iguales la aceptación de un mensaje permisivo o punitivo, respecto a la cocaína y el éxtasis, los jóvenes consideran que es más beneficioso para ellos un mensaje punitivo ( 84,3 y $80 \%$ para el éxtasis y la cocaína respectivamente), les resulta más creíble $(70$ y $71,4 \%)$ y están más dispuestos a seguirlo $(65,7$ y $74,3 \%)$. Hasta aquí todo parece indicar que los mensajes punitivos pueden ser, en términos generales, mejor aceptados que los permisivos, sobre todo para drogas como el éxtasis y la cocaína, y que, tal vez, con una insistencia suficiente puedan decantar las actitudes de los jóvenes hacia la droga en una dirección marcadamente negativa.

No obstante, vamos a contemplar este tema desde otro punto de vista. Así, vamos a presentar los porcentajes de respuestas a estas mismas preguntas de dos subgrupos de la muestra. En el CUADRO 3 se presentan los resultados para el grupo de no consumidores y consumidores experimentales del pasado de no más de 3 ocasiones (no consu- 
midores-NC) por una parte, y para el grupo de consumidores actuales o del pasado que consumen o han consumido más de un año (consumo frecuente-CF). Respecto a la preferencia de los mensajes, sólo consideraremos en este caso la categoría PERMISIVO, ya que, al tratarse de una elección forzada de dos alternativas, es suficiente con presentar los resultados de una categoría.

CUADRO 3: Actitudes ante la droga y los mensajes para el grupo de no consumidores (NC) y consumidores frecuentes (CF) (en \%).

\begin{tabular}{|lcc|}
\hline & $\mathrm{NC}$ & $\mathrm{CF}$ \\
La droga es mala & 76 & 15 \\
La droga es buena & 0 & 0,3 \\
La droga no es buena ni mala & 0 & 0 \\
Depende de la droga & 0,3 & 24 \\
Depende de cómo se utilice & 20 & 57 \\
Ayuda a mejorar las relaciones & 0 & 15 \\
Es muy peligrosa para la salud & 88 & 48 \\
Debería penalizarse su consumo & 23 & 0 \\
Es difícil controlar su consumo & 60 & 36 \\
Debería estar legalizada & 0,8 & 54 \\
Quien consume es que no sabe divertirse sin droga & 52 & 15 \\
Hace que te lo pases mejor con los amigos & 12 & 21 \\
(SÓLO PERMISIVO) & & \\
MENSAJES CANNABIS & $\mathrm{NC}$ & $\mathrm{CF}$ \\
1. Interesante & 19 & 81 \\
2. Dispuesto a seguir & 0,4 & 84 \\
3. Beneficioso & 0,4 & 66 \\
4. Más cré́ble & 12 & 75 \\
5. Te identificas & 12 & 81 \\
6. Dar a un amigo que consume & 12 & 72 \\
MENSAJES EXTASIS & & \\
1. Interesante & 38,4 & 57 \\
2. Dispuesto a seguir & 0,7 & 54 \\
3. Beneficioso & 0 & 21 \\
4. Más créble & 0,7 & 45 \\
5. Te identificas & 11,5 & 60 \\
6. Dar a un amigo que consume & 23 & 57 \\
MENSAJES COCAÍNA & & \\
1. Interesante & 38 & 57 \\
2. Dispuesto a seguir & 0,3 & 48 \\
3. Beneficioso & 0,38 & 36 \\
4. Más creíble & 0,3 & 51 \\
5. Te identificas & 0,3 & 54 \\
6. Dar a un amigo que consume & 0,7 & 51 \\
\hline
\end{tabular}


Estos resultados nos dan, desde luego, otra visión del tema. Así, mientras la actitud de partida hacia la droga es claramente negativa para los NC, es justo lo contrario para los $\mathrm{CF}$, quienes, aunque reconocen que es peligrosa para la salud $(48 \%)$, piensan que debería legalizarse $(54 \%)$. Una parte de ellos reconoce que la droga sirve para mejorar las relaciones (15\%) o para pasarlo mejor con los amigos $(21 \%)$. En cuanto a la actitud ante los mensajes, los resultados muestran unas diferencias muy evidentes y claras entre los NC y los CF. Los consumidores frecuentes aceptan mejor que los no consumidores el mensaje permisivo (de reducción de daño) que el punitivo para todas las drogas, pero muy especialmente para el cannabis, donde la credibilidad del mensaje permisivo es muy significativa (75 y $12 \%$ para los CF y NC respectivamente). Esto nos hace reflexionar sobre la conveniencia de emplear determinados mensajes en los programas de prevención sin considerar el tipo de droga al que se refieren ni al tipo de jóvenes al que va dirigido. Así, en el caso de la droga que más nos interesa en este estudio, el cannabis, mientras un $96 \%$ de los no consumidores está dispuesto a seguir un consejo punitivo, un $84 \%$ de los consumidores frecuentes están dispuestos a seguir un consejo permisivo con el consumo y de reducción de daño. La diferencia es espectacular. Es difícil pensar que por mucho que se repitan los mensajes punitivos en un programa de prevención, va a cambiar sustancialmente la actitud ante la droga de los consumidores actuales. Es posible también que los no consumidores, con una actitud negativa hacia la droga de partida, no necesiten ser expuestos en demasiadas ocasiones a mensajes negativos sobre las drogas para no consumir. Dicho de otro modo, es posible que los programas de prevención sólo influyan sobre aquellos que ya tienen una actitud negativa y que, de todas formas, no presentan riesgo de consumo futuro, y que, por otra parte, no influyan sobre aquellos que consumen en la actualidad. Ahora bien, los defensores de los programas de prevención argumentarán que el cambio de actitud no es la única vía, ni siquiera la más importante, para conseguir un cambio en el consumo, sino que el entrenamiento en habilidades para resistirse a la presión de los pares a consumir, será lo más eficaz. Ahora bien, hay que contemplar otra cuestión al respecto, y es que entre los jóvenes tiene lugar un proceso de selección interpersonal de amigos con conductas y valores similares (Kandel, 1978a). O sea, que en muchos casos esta selección de amigos con actitudes similares ante la vida, entre las que pueden encontrarse las relativas al consumo de drogas, sería previo al consumo y formaría parte de actitudes y susceptibilidades más arraigadas que las relativas a la droga y que serían especialmente resistentes al cambio. Sigue sin quedar claro que la orientación teórica y práctica de los actuales programas de preven- 
ción del consumo de drogas, tengan un resultado de mayor alcance de lo que supone "convencer a los ya convencidos".

Pero, para seguir este argumento, es necesario realizar más análisis que nos orienten al respecto. Así, si existen diferencias fundamentales entre los dos grupos ( $\mathrm{NC}$ y $\mathrm{CF}$ ) en dimensiones de personalidad, y si estas dimensiones tienen alguna relevancia en cuanto a determinar el consumo y abuso de drogas, o en cuanto a la aceptación de un determinado tipo de mensajes sobre la droga, se confirmaría nuestro argumento de que "se convence a los convencidos". Pero veamos estos resultados.

\subsection{Diferencias en personalidad entre los no consumidores (NC) y los consumidores frecuentes $(\mathrm{CF})$}

Hemos contrastado las medias de las dimensiones de personalidad del cuestionario EPQ (Extraversión, Neuroticismo y Psicoticismo) además del factor Sinceridad de los dos grupos anteriores, pero añadiendo una variante. Así, hemos comparado el grupo $\mathrm{CF}(\mathrm{N}=33)$ con el grupo NC pero excluyendo a los consumidores experimentales de cannabis para aislar cualquier efecto del consumo. Seguiremos llamando a este grupo $\mathrm{NC}(\mathrm{N}=16)$ y sólo cuando se considere oportuno se hará la distinción necesaria. Se han obtenido dos diferencias estadísticamente significativas en $P(t=-2.76, p<.01)$ y en $S(t=-5.16$, $\mathrm{p}<.001$ ). La diferencia en Psicoticismo es esperable en cuanto a que los aspectos que componen tal dimensión (impulsividad, agresividad, búsqueda de sensaciones, etc.) están asociados tradicionalmente con el consumo de drogas. Ahora bien, lo que resulta más llamativo es la diferencia en Sinceridad a favor de los consumidores de cannabis. La sinceridad no parece ser un rasgo característico de los consumidores de droga, pero en nuestro estudio es una característica claramente definitoria de los mismos. ¿Cómo podemos interpretar este resultado? Si bien no existe investigación ni consenso suficiente sobre el significado de esta variable, existen indicios de que se trata de un rasgo estable de personalidad, aunque no se sabe muy bien cuál (Eysenck y Eysenck, 1975). Se ha apuntado la posibilidad de que se trate de un factor que mida la no asunción de reglas sociales (Eysenck y Eysenk, 1964), ya que sus ítems se refieren a negligencias en cuestiones que atañen las normas sociales. Si este fuera el caso, sería más adecuado denominar a este factor Inconformidad, haciendo referencia más al no convencionalismo, más que a Sinceridad. Si bien no existe en la investigación general suficiente evidencia para lo que aquí estamos proponiendo, sí es cierto que la validación de este factor puede provenir de estudios como éste, por lo que en principio aceptare- 
mos este razonamiento e interpretación del factor $\mathrm{S}$, al que denominaremos I (inconformismo).

\subsection{Predictores del consumo actual de cannabis}

Si estas dos dimensiones ( $\mathrm{P}$ e I) distinguen a los no consumidores y consumidores frecuentes, es importante considerar si tienen alguna implicación en la conducta de consumir cannabis y en la dependencia y abuso del mismo. Así, se han realizado numerosos análisis de regresión teniendo como variables dependientes el consumo actual de cannabis y la categoría de dependencia y abuso, y como variables independientes las dimensiones de personalidad, introduciendo en posteriores análisis variables circunstanciales y situacionales (calidad de vida, número de problemas y emocionalidad negativa), y actitudes hacia la droga. Todas las regresiones son logísticas, ya que las variables dependientes son dicotómicas y en todos los análisis, la introducción de las variables se realizó "paso a paso". A continuación resumiremos los resultados obtenidos.

En un primer análisis se incluyeron como variables independientes las tres dimensiones de personalidad eysenckianas. $\mathrm{P}$ y $\mathrm{E}$ fueron incluidas en la ecuación, pero $\mathrm{E}$ no resultó significativa al nivel $\mathrm{p}<.05$. Para la variable $\mathrm{P}, \mathrm{B}=.20(\mathrm{p}<.05)$. Cuando se añadió a las dimensiones de personalidad la variable I, ésta fue la única que se incluyó en la ecuación $(\mathrm{B}=.24, \mathrm{p}<.01)$ dejando fuera de la ecuación a las demás. Podemos interpretar de estos resultados que una dimensión de personalidad como $\mathrm{P}$ es buen predictor del consumo actual, pero que el Inconformismo es un predictor mucho más importante del consumo actual.

$\mathrm{Si}$ a las dimensiones de personalidad y al Inconformismo añadimos la variable ACTITUD, obtenemos otra combinación de variables predictoras. Así, la variable ACTITUD es la que se incluye en la ecuación en el primer paso (como esta variable es categórica sólo indicaremos el nivel de significación, $\mathrm{p}<.05)$, seguidas por $\mathrm{I}(\mathrm{B}=.27, \mathrm{p}<.05)$ y por $\mathrm{E}(\mathrm{B}=.25, \mathrm{p}<.05)$. Por tanto, en la predicción del consumo actual de cannabis influye especialmente la actitud que se tenga ante la droga, pero también la actitud general frente a la vida y la sociedad (Inconformismo) y la personalidad (Extraversión). Si los programas de prevención pretenden cambiar las actitudes hacia las drogas con mensajes negativos, es más difícil que consigan cambios de mentalidad a otros niveles o de personalidad, aspectos que, como vemos, están relacionados con el consumo de cannabis. En relación con esto, en un análisis logístico en el que añadimos a las variables anteriores las de CALIDAD DE VIDA, PROBLEMAS y EMOCIONALIDAD 
NEGATIVA, observamos que ninguna de ellas fue incluida en la ecuación, lo que indica que la conducta de consumir cannabis tiene motivos más estables y no transitorios.

Estos resultados se confirman si prescindimos en el análisis de ACTITUD e incluimos las siete actitudes específicas que más arriba enunciamos (esto lo hacemos así para evitar posible colinealidad).

En este caso, 4 variables son incluidas en la ecuación (I, E, DROGAS 5 y DROGAS 6, estas dos últimas categóricas). Las únicas con $\mathrm{B}$ significativas al nivel $\mathrm{p}<.05$ son Inconformidad $(\mathrm{B}=.34)$ y "Quien consume es que no sabe divertirse sin drogas" (B negativa). Próximas a la significación $(\mathrm{p}<.06)$ están Extraversión y "Debería estar legalizado".

Es decir, se confirma que en el consumo actual de cannabis influyen aspectos de personalidad ( $\mathrm{P}$ y $\mathrm{E}$ ), de actitud general frente a la sociedad (I) y especialmente frente a la droga. Al respecto de esto último, el consumo está asociado con una actitud más favorable hacia la droga, la impresión de que su consumo es una forma más de diversión pero que no cubre todas las formas de diversión (recordemos la $B$ negativa) y que debería estar legalizada. Aparte de la influencia de los demás factores, parece que este tipo de variables forman parte de un sistema de valores amplio y de la comprensión general de la sociedad y las relaciones humanas y no parecen, desde luego, susceptibles de cambio salvo ímprobos esfuerzos de las autoridades sanitarias y políticas.

\subsection{Predictores de la dependencia, abuso y consumo compulsivo de cannabis}

En este grupo de análisis hemos querido constatar si la personalidad y algunos aspectos más transitorios como la cantidad de problemas que uno tiene en la actualidad o la calidad de vida, predicen la dependencia, el abuso o la compulsión del cannabis. Las variables dependientes representan el sumatorio de las respuestas afirmativas a los criterios del DSM-IV, y por tanto no son variables categóricas (dependencia/no dependencia, etc). Lo hemos hecho así para hacer valer la riqueza de información que teníamos al respecto.

$\mathrm{Si}$ consideramos como variables independientes las dimensiones de personalidad y el Inconformismo, obtenemos que el Psicoticismo es la única variable incluida en las ecuaciones, tanto para la dependencia (beta $=.39, \mathrm{p}<.001$ ), como para abuso (beta $=.40, \mathrm{p}<.001$ ) y para compulsión (beta $=.46, \mathrm{p}<.001$ ), con una varianza explicada del 12 , 16 y $21 \%$, respectivamente. 
Si añadimos las variables PROBLEMAS y CALIDAD DE VIDA, aparecen algunas variaciones. Así, mientras que para el abuso, $P$ es la única variable incluida (beta $=.40, \mathrm{p}<.001$ ) (varianza explicada del $16 \%$ ), para la dependencia, la ecuación incluye las variables PROBLEMAS (beta=.35, $\mathrm{p}<.01$ ) e Inconformismo (beta=.28, $\mathrm{p}<.05$ ) (varianza explicada del 24\%), y para la compulsión las variables $\mathrm{P}$ (beta $=3.28, \mathrm{p}<.01$ ) y PROBLEMAS (beta=.27, $\mathrm{p}<.05$ ) (varianza explicada del 28\%).

Así, se comprueba que mientras la cantidad de problemas y su impacto negativo son buenos predictores de la dependencia y el consumo compulsivo de cannabis, también es cierto que factores más estables como el Psicoticismo y el Inconformismo se relacionan claramente.

\subsection{Predictores de la aceptación de mensajes permisivos sobre el consumo de cannabis}

Una vez hemos visto los mejores predictores del consumo y abuso del cannabis, entre los cuales hay factores estables de personalidad, vamos a realizar el mismo tipo de análisis de regresión múltiple tomando como variable dependiente CAMEP. Esta variable es el sumatorio de todas las respuestas afirmativas de preferencia a los mensajes permisivos a partir de las 6 preguntas sobre los dos mensajes del cannabis que se les presentan a los jóvenes de este estudio, y por lo tanto es una variable continua.

Cuando se utilizan como variables independientes las 3 dimensiones eyxenckianas, sólo la $\mathrm{P}$ es incluida en la ecuación (beta=.26, $\mathrm{p}<.05$ ), la cual explica tan sólo el $0,7 \%$ de la varianza. El Inconformismo es una variable mejor predictora que las de personalidad, ya que, al añadirla a las anteriores, es la única incluida en la ecuación (beta $=.41$, $\mathrm{p}<.001$ ), con una varianza explicada del $16 \%$.

$\mathrm{Si}$ a las anteriores variables añadimos CANAH (consumo de cannabis en la actualidad) y CANAL (consumo de cannabis en el pasado pero interrumpido en el presente), son estas dos variables las únicas incluidas en la ecuación, explicando un $45 \%$ de la varianza.

Si además añadimos las 7 actitudes específicas, las únicas variables incluidas en la ecuación son el consumo actual y pasado de cannabis y "Debería estar legalizado", con una varianza explicada del $49 \%$.

Podemos concluir que, aunque las variables de personalidad y el Inconformismo predicen en alguna medida la aceptación de mensajes permisivos del consumo de cannabis, es el hecho de consumir (tanto 
en el presente como en el pasado) y la actitud favorable a la legalización, los mejores predictores. En el CUADRO 4 se resumen los resultados que acabamos de exponer, de forma que se integren y ordenen los datos a partir de los distintos análisis de regresión con diferentes bloques de variables independientes, y de aquellos análisis que cuentan con todas las variables independientes de una vez.

CUADRO 4: Principales predictores del consumo, dependencia, abuso, compulsión y aceptación de un mensaje positivo sobre el consumo de cannabis.

PREDICTORES (por orden de importancia)

\begin{tabular}{|c|c|}
\hline \multirow[t]{4}{*}{ CONSUMO ACTUAL } & $\begin{array}{l}\text { Actitud general } \\
\text { Inconformismo } \\
\text { Actitud específica (negativa): "Quien consume es que } \\
\text { no sabe divertirse sin } \\
\text { drogas" }\end{array}$ \\
\hline & Psicoticismo \\
\hline & Extraversión \\
\hline & Actitud específica: "Debería estar legalizada" \\
\hline \multirow[t]{3}{*}{ DEPENDENCIA } & Problemas \\
\hline & Inconformismo \\
\hline & Psicoticismo \\
\hline ABUSO & Psicoticismo \\
\hline \multirow[t]{2}{*}{ COMPULSIÓN } & Psicoticismo \\
\hline & Problemas \\
\hline \multirow[t]{5}{*}{ MENSAJE PERMISIVO } & Consumo actual de cannabis \\
\hline & Consumo pasado de cannabis \\
\hline & Actitud específica: "Debería estar legalizada" \\
\hline & Inconformismo \\
\hline & Psicoticismo \\
\hline
\end{tabular}

En este cuadro podemos destacar la gran importancia de la actitud general hacia las drogas y de actitudes específicas a la hora de explicar el consumo actual de cannabis y la aceptación de mensajes permisivos sobre su consumo. También hay que destacar que las variables de personalidad (especialmente P) e I juegan un importante papel en este caso. Hay también que señalar en este punto que las dos variables (Psicoticismo e Inconformismo) están muy relacionadas en nuestra muestra $(\mathrm{r}=.43, \mathrm{p}<.001)$. Por otra parte, la cantidad de problemas que uno tenga y el hecho de consumir o haber consumido cannabis, tiene valor predictivo de la dependencia/compulsión y de la aceptación de mensajes permisivos, respectivamente. Por último, el Psicoticismo, factor estable de la personalidad, es un predictor importante de la dependencia y abuso del cannabis y de su consumo compulsivo $\mathrm{y}$, por tanto, poco controlado. 


\section{IV.LOS PROGRAMAS DE PREVENCIÓN Y EL PROBLEMA DE LA DROGA}

Si una serie de variables de personalidad (P y E), y actitudes frente a la sociedad (I) y frente a las drogas (favorable a la droga y a su legalización) son los mejores predictores del consumo, abuso, y aceptación de mensajes permisivos y de reducción de daños respecto al cannabis, es fácil comprender cómo los programas de prevención han resultado, en general, un fracaso respecto al consumo general de drogas y en particular de cannabis. Como decíamos más arriba, parece ser que aumentar la duración y la intensidad de los programas mejoraría su eficacia. Pero tampoco está claro que esto sea así. Además, esto encarecería todavía más la aplicación de estos programas. La aplicación del DARE en Estados Unidos es de 100 dólares por estudiante (Dukes, Ullman y Stein, 1996). Esto nos lleva a plantearnos si tiene sentido mantener la existencia de estos programas, al menos tal como están enfocados en la actualidad. Y esto, a su vez, nos lleva a plantearnos la posibilidad de proponer un cambio en la política de drogas hacia la no intervención del Estado. Esta propuesta no tiene visos de ser bien aceptada por la opinión pública debido al otro mito del que se hablaba al principio de este artículo: el mito del problema de la dro$\mathrm{ga}$. Este mito puede ser expuesto como la creencia firme e incontestada de que la droga es un gran problema social y sanitario en todos los países, y que hay que erradicar, empezando por convencer a los jóvenes que no consuman. En este apartado hablaremos del problema de la droga a partir de los resultados que hemos obtenido en nuestra muestra, y referidos al cannabis. No es posible generalizar con facilidad a las demás drogas, ni es posible hacerlo a partir de estos resultados. No obstante, la pretensión de mostrar estos resultados es abrir un diálogo que permita realmente la superación del "problema de la droga". Veamos.

Vamos a detenernos en el grupo de CF (consumidores frecuentes). En el CUADRO 5 se presentan las pautas de consumo de cannabis de los jóvenes de nuestra muestra, así como el control percibido del consumo, los efectos percibidos a corto y largo plazo, niveles de dependencia y abuso y las motivaciones del consumo.

Entre los resultados más destacables presentados en el CUADRO 5 encontramos que las pautas de consumo son muy variadas. Así, un $18 \%$ mantienen un consumo ocasional, mientras que el $27 \%$ consumen muchas veces en situaciones concretas (fiestas, etc.) y el $33 \%$ consumen casi todas las veces en esas situaciones concretas. Un $18 \%$ consumen muchos días al margen de esas situaciones concretas, y aunque este consumo no sea necesariamente diario, lo hemos denomina- 
CUADRO 5: Pautas de consumo de cannabis, y otros aspectos relacionados (resultados en porcentajes)

$\begin{array}{lc}\text { Pautas de consumo } & \\ \text { Consumo ocasional } & 18 \\ \text { Consumo circunstancial } & 27 \\ \text { Consumo habitual } & 33 \\ \text { Consumo "a diario" } & 18 \\ \text { Situaciones de mayor consumo } & \\ \text { Discoteca } & 39 \\ \text { Fiesta } & 90 \\ \text { Con la pareja } & 33 \\ \text { Sólo } & 24 \\ \text { Mezclar con otras drogas } & \\ \text { Nunca } & 0,6 \\ \text { Alguna vez } & 33 \\ \text { Frecuentemente } & 27 \\ \text { Siempre } & 33 \\ \text { Sustancias que mezcla } & \\ \text { Alcohol } & 93 \\ \text { Anfetaminas } & 0,6 \\ \text { Tranquilizantes } & 0 \\ \text { Alucinógenos } & 15 \\ \text { Heroína } & 0 \\ \text { Cocaína } & 21 \\ \text { Extasis } & 27 \\ \text { Tiag } & \end{array}$

Diagnóstico de dependencia y abuso según criterios del DSM-IV Dependencia 45

Abuso 57

Control percibido del consumo de cannabis

$\begin{array}{lc}\text { Nada } & 0,9 \\ \text { Algo } & 12 \\ \text { Bastante } & 12 \\ \text { Mucho } & 36 \\ \text { Totalmente } & 30\end{array}$

Efectos a corto plazo del cannabis Bienestar

\begin{tabular}{ccc|} 
Nunca & A menudo & Siempre \\
0,3 & 69 & 27 \\
45 & 48 & 0,6 \\
0,9 & 54 & 33 \\
18 & 45 & 36 \\
30 & 39 & 30 \\
30 & 48 & 21 \\
39 & 33 & 27 \\
56 & 40 & 0,3 \\
50 & 25 & 25 \\
40 & 46 & 12
\end{tabular}

Euforia

Risas

Sedación

Letargia

\section{5}

7




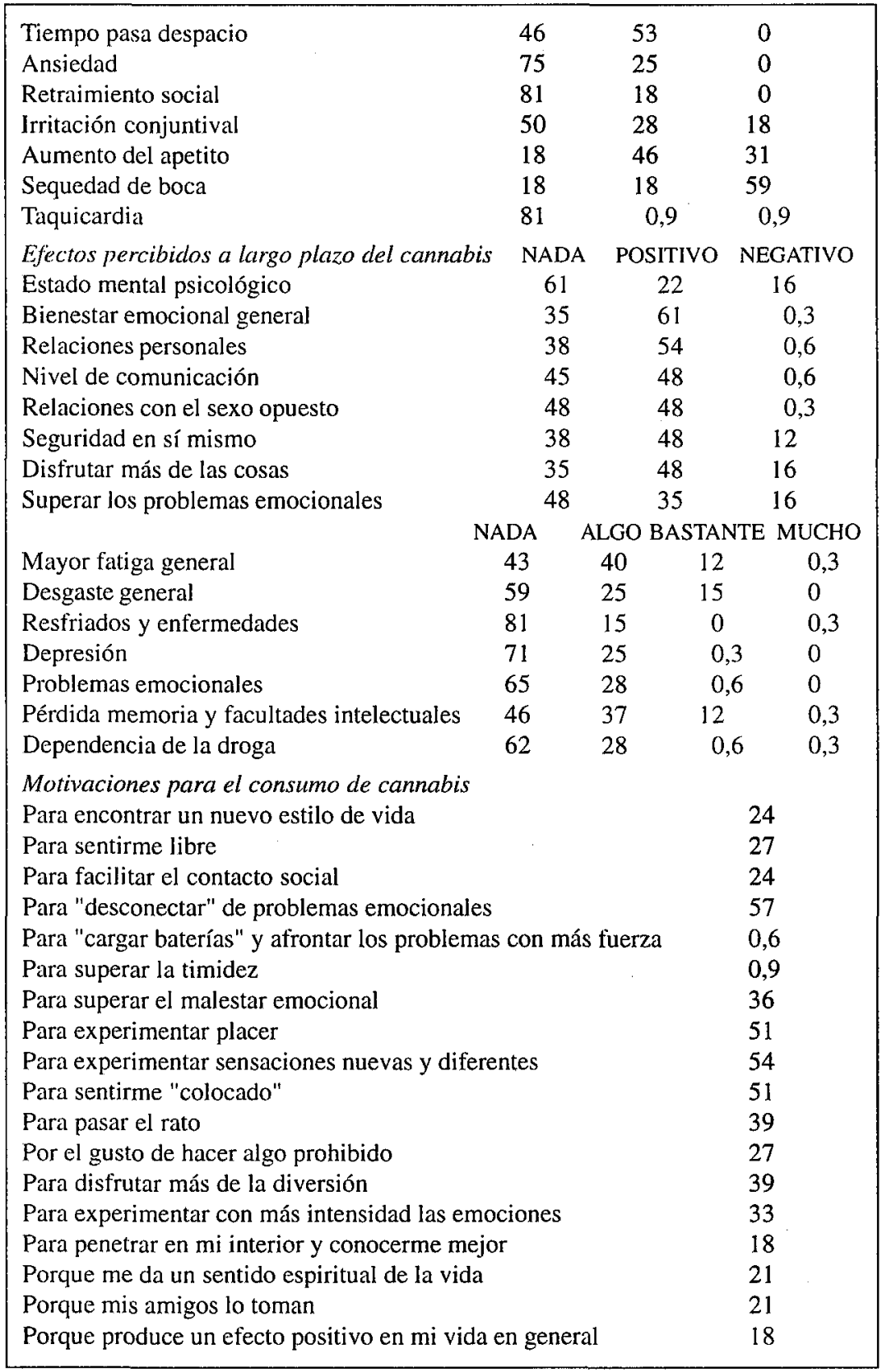


do "a diario". Ya que la muestra, como dijimos anteriormente, se ha obtenido a partir de la búsqueda de personas que resaltaban por su consumo, cabe esperar que esté sesgada hacia una mayor frecuencia de consumo de lo que encontraríamos en una muestra representativa de la población general de consumidores. No obstante estas matizaciones, que servirán para la interpretación del resto de los resultados de este apartado, cabe decir que la mayor frecuencia de consumo se da en situaciones concretas. Esto se confirma al ser la situación de fiesta donde más se da el consumo $(90 \%)$ con mucha diferencia de las demás y sobre todo del consumo en solitario (24\%), que se presenta en una menor proporción. Si además de un alto control percibido del consumo (el $36 \%$ perciben mucho control y el $30 \%$ total control), el tener reglas para el cuándo y dónde consumir drogas (consumo en situaciones concretas) es un reflejo del control de la misma (ver Cohen y Sas, 1992, para el consumo de cocaína), podríamos concluir que un porcentaje alto de los consumidores de nuestra muestra controlan en buena medida el consumo.

No obstante, los datos sobre los porcentajes de jóvenes con diagnóstico de dependencia (45\%) y de abuso (57\%) no nos hablan precisamente de control de la droga. Ahora bien, a lo largo del estudio y hablando con los consumidores de cannabis, tuvimos la impresión de que los criterios diagnósticos del DSM-IV eran demasiado laxos. Dicho de otra forma, de que "hinchan" la proporción de dependientes y de consumidores que abusan en la población. Para comprobar esto hicimos una prueba. Separamos a los consumidores "a diario" de cannabis $(\mathrm{N}=7)$ y contrastamos las medias de los sumatorios de dependencia y abuso con el resto de los consumidores $(\mathrm{N}=23)$. Obtuvimos una diferencia estadísticamente significativa en Dependencia $(\mathrm{t}=-$ $2.45, \mathrm{p}<.05)$ pero no en Abuso. Así, si consideramos como variable para el contraste no el diagnóstico de dependencia, sino el sumatorio de los puntajes en respuesta a los criterios del DSM-IV, existen diferencias significativas entre los que tienen una pauta de consumo "controlada" y los que consumen a diario en cuanto a la dependencia de la droga. Si bien este resultado debe ser replicado en posteriores investigaciones con muestras mayores, es indicativo de que el DSM-IV puede estar empleando criterios "laxos" para el diagnóstico de dependencia y abuso.

Por otra parte, hay que resaltar que los efectos a corto plazo apenas presentan situaciones de riesgo o peligro. Si bien se presentan algunos inconvenientes en las funciones mentales, con un $27 \%$ señalando dificultad en los procesos mentales complejos y un $25 \%$ percepciones sensoriales distorsionadas, un $75 \%$ nunca presentan problemas de ansiedad y un $81 \%$ nunca presentan retraimiento social. 
Por otra parte, los efectos percibidos a largo plazo son positivos en mucha mayor medida que negativos. Así, la mayor parte de los efectos negativos (mayor fatiga general, desgaste general, etc.) o bien no se presentan como consecuencias a largo plazo del consumo o bien aparecen en poca medida (algo). Ninguno de estos efectos negativos supera la proporción del $15 \%$ en cuanto a que representen una consecuencia bastante negativa del consumo. En cuanto a los efectos del primer bloque (estado mental psicológico, bienestar emocional general, etc.) hay que resaltar que, aunque cerca de la mitad de los consumidores no percibe ningún tipo de efecto a largo plazo, si comparamos las consecuencias positivas con las negativas, el consumo sostenido de cannabis ha tenido un impacto escasamente negativo y claramente positivo, para más de la mitad de los consumidores en bienestar emocional general $(61 \%)$ y relaciones personales $(54 \%)$ y en un $48 \%$ de los consumidores en el nivel de comunicación, relaciones con el sexo opuesto, seguridad en sí mismo y disfrutar más de las cosas. Hay que resaltar que el $35 \%$ reconocen un impacto positivo del consumo continuado respecto a la superación de los problemas emocionales, si bien un $16 \%$ ha respondido en sentido contrario.

En cuanto a las motivaciones para el consumo, hay que destacar las referentes a la búsqueda de sensaciones y diversión, es decir, al aspecto lúdico del consumo de droga, como son: experimentar placer $(51 \%)$, experimentar sensaciones nuevas y diferentes $(54 \%)$, sentirse "colocado" (51\%) y disfrutar más de la diversión (39\%). Ahora bien, el resultado más llamativo es el que se refiere al consumo para "desconectar" de problemas emocionales (57\%). Esto puede significar que más de la mitad de los consumidores se drogan para no afrontar los problemas, para huir de ellos, lo que constituiría un comportamiento inmaduro y de evitación. Ahora bien, esto no está claro que sea así. Veamos. Por otra parte, en una Encuesta Nacional sobre Drogas en Estados Unidos (Timmer, Veroff y Colten, 1985) se concluye que la "relajación" de los problemas que proporciona la droga no es incompatible con otras estrategias activas de coping, como el recurso a la red social y la comunicación, y que, más bien al contrario, las personas con mejor ajuste psicológico son las que consumen drogas de forma moderada frente a las que no consumen nada, que tienen peor ajuste. El consumo excesivo está relacionado con la necesidad de "escapar" a unos niveles altos de estrés y es característico de personas con mal ajuste psicológico, mientras que el consumo moderado resulta saludable como un recurso para "relajarse" en las situaciones de estrés moderado de la vida diaria. También aquellas personas con una mayor vulnerabilidad al estrés, que sienten que las situaciones de tensión sobrepasan sus capacidades de afrontamiento y que poseen una 
baja autoconfianza, son más propensas a consumir excesivamente para afrontar el estrés. Por otra parte, en algunos estudios sobre coping y drogas (Wills, 1985) se concluye que la Distracción (que equivale a nuestra variable "desconectar" de problemas) es una válida y eficaz estrategia para afrontar el estrés. En definitiva, el coping está relacionado de una forma particular con el nivel de estrés y el consumo de drogas. Así, a niveles moderados de estrés, el coping incrementa el consumo de drogas, mientras que con niveles altos de estrés aumenta el consumo de drogas mientras se reduce el coping. Así, el nivel de estrés sería una variable moduladora de la relación entre coping y consumo de drogas. $\mathrm{El}$ hecho de que estas variables pueden covariar positivamente lo muestra el hecho, constatado por Wills, de que el apoyo social está asociado positivamente con el fumar. Cuanto más dependía un estudiante de su grupo de pares para hablar de sus problemas, más involucrado estaba en la conducta de fumar tabaco. Este mismo autor encontró que si bien un factor general de autoestima y de control percibido correlacionaron negativamente con el consumo de tabaco y alcohol, dos subescalas de ambos constructos, autoconfianza social y control percibido en situaciones sociales, correlacionaron significativamente $(\mathrm{p}<.01)$ con el consumo de alcohol y con el consumo de tabaco y alcohol, respectivamente. Esto da a entender que el consumo de drogas (en este caso legales) puede estar positivamente relacionado con el apoyo social y el incremento de los aspectos sociales de la autoestima y el control percibido.

$\mathrm{Si}$ en nuestra muestra contrastamos las medias de los modos de afrontamiento, que son las Estrategias activas cognitivas (EAC), Estrategias activas comportamentales (EACO) y Estrategias de evitación (EE) entre los consumidores de cannabis actuales $(N=33)$ y el resto de la muestra que no consume en la actualidad ninguna droga ilegal $(\mathrm{N}=38)$ no encontramos diferencias estadísticas en las estrategias activas y sí un mayor empleo significativo de las estrategias de evitación entre los consumidores actuales $(p<.05)$. Hay que destacar que en el caso de las EACO, los consumidores actuales las emplean más hasta un punto que se acerca a la significación $(\mathrm{p}<.08)$. Si bien no hay diferencias significativas entre los dos grupos en cuanto a cantidad de problemas, sí es cierto que en dos subescalas de calidad de vida (relaciones emocionales e involucración en el trabajo), los consumidores actuales se sienten más insatisfechos. Ahora bien, si comparamos a los consumidores actuales de cannabis con los no consumidores de ninguna droga ilegal en la vida $(\mathrm{NC}=16)$, encontramos los mismos resultados en los modos de afrontamiento que anteriormente hemos citado, pero además vemos que los consumidores actuales tienen más problemas en general $(\mathrm{p}<.05)$ y en particular más problemas 
económicos $(p<.01)$, de vivienda $(p<.01)$ y familiares $(p<.05)$. Además, se sienten más insatisfechos en las relaciones emocionales $(\mathrm{p}<.05)$, familiares $(\mathrm{p}<.01)$, en el área económica $(\mathrm{p}<.05)$ y se apoyan menos en la religión $(\mathrm{p}<.01)$. Podemos ver que en aquellas áreas donde más problemas tienen los consumidores actuales, son las que más insatisfacción encuentran, si bien no hay diferencias significativas en el índice general de calidad de vida ni en felicidad. Estos resultados pueden interpretarse en la línea de los estudios citados sobre coping y consumo de drogas. Así, por una parte, la diferencia significativa en el volumen de problemas pude ocasionar en los consumidores actuales un nivel alto de tensión al que responden con un mayor consumo y más estrategias de evitación. Por otra parte, no utilizan menos estrategias activas que los no consumidores, con lo cual se hace patente que es compatible el uso de estas estrategias con el consumo de drogas. De hecho, para el total de la muestra, los problemas se relacionan significativamente con los 3 tipos de estrategias $(p<.001$ para EAC y $\mathrm{p}<.01$ para EACO y EE). O sea, que cuantos más problemas, más estrategias de afrontamiento se ponen en marcha, tanto de evitación como activas. El que muchos consumidores (57\%) informan del consumo de cannabis como una forma de "desconectar" de los problemas no representa necesariamente una estrategia de huida, sino que, como ya se ha señalado antes, puede representar una válida estrategia de afrontamiento de niveles altos de estrés.

\subsection{La hipótesis de la escalada de drogas}

Si el consumo de cannabis no produce un efecto tan negativo en los jóvenes sobre su salud mental como pudiera pensarse en un principio, el mayor peligro que muchos ven en este consumo, es que lleve inevitablemente al consumo y abuso de otras drogas ilegales y peligrosas (cocaína y heroína principalmente). Es lo que se llama la hipótesis de la escalada de las drogas. En una revisión de la evidencia al respecto, Kandel et al. (1992) concluyen que si bien los consumidores de "drogas duras" empezaron mayoritariamente consumiendo tabaco, alcohol y cannabis, esto no quiere decir que los que consumen cannabis vayan a progresar en la dirección del consumo de drogas más peligrosas. Así, el consumo de cannabis es una condición necesaria pero en modo alguno suficiente para el paso a otras drogas.

Por otra parte, con datos del National Household Surveys (Fishburne et al., 1980), se informa que casi todos los jóvenes entre 18-25 años que son consumidores de cannabis lo son de tabaco y alcohol, pero poco más de una cuarta parte de ellos ha probado cualquier otra droga ilegal. Muy pocos de los que han probado otras drogas son consumidores actuales de las mismas. 
En un estudio muy reciente de consumidores de cannabis de Amsterdam, Cohen y Sas (1996) argumentaron que la teoría de la escalada se confirmaría si el 75\% o más de los que consumen cannabis, presentan algún consumo de otras drogas como la heroína, la cocaína y el éxtasis. En su muestra, en ningún grupo de consumidores de cannabis, ni siquiera en los consumidores "fuertes" se constató un consumo de cualquiera de estas tres drogas que alcanzara la proporción indicada.

La muestra de nuestro estudio es especialmente interesante al respecto, ya que debido al sistema de búsqueda, que anteriormente explicamos, cabría esperar que muchos de los consumidores de cannabis consumieran también otras drogas ilegales. Si sumamos el consumo actual con el consumo pasado interrumpido, independientemente del nivel del mismo, obtenemos que de la muestra de consumidores actuales ( $\mathrm{N}=33$ ), el $66 \%$ consume o ha consumido cocaína (de los cuales el $51 \%$ ya la han dejado), el $51 \%$ éxtasis (el $36 \%$ ya lo han dejado) y el $0,3 \%$ heroína. Así, en una muestra de consumidores de cannabis favorable para encontrar confirmación de la hipótesis de la escalada, si nos atendemos a la operacionalización al respecto establecida por Cohen y Sas (1996), se desconfirma tal hipótesis. Así, parece que el cannabis no resulta tan peligroso para la juventud en el sentido de conducirles necesariamente al consumo de drogas "duras".

\section{DISCUSIÓN}

El estado actual de conocimientos sobre la eficacia de los programas de prevención del consumo de drogas muestra, en el mejor de los casos, inconsistencias, cuando no resultados desalentadores. En este estudio se han puesto de manifiesto algunas posibles razones que explican este aparente fracaso. Así, se ha constatado la importancia de factores estables de personalidad (especialmente Psicoticismo) y de actitud frente a la vida y la sociedad (Inconformismo) como determinantes del consumo y abuso de cannabis. Además, los consumidores frecuentes de cannabis muestran una preferencia muy marcada por aquel tipo de mensajes y consejos permisivos sobre el consumo de drogas en general y del consumo de cannabis en particular, resultándoles a la mayoría más interesantes $(81 \%)$, creíbles $(75 \%)$ y a los que están más dispuestos a seguir (84\%), frente a los mensajes antidroga, más aceptados mayoritariamente por los no consumidores. Así, es posible que los programas de prevención estén infravalorando la importancia de los factores estables, como el Psicoticismo e Inconformismo, como factor de resistencia a aceptar estos programas por los 
consumidores de drogas, y que la insistencia en mensajes negativos y anti-droga esté resultando ineficaz con los consumidores (al menos de cannabis, que constituyen la principal representación de nuestra muestra), y que sólo convenzan a los "ya convencidos", es decir, que influyan en la reducción del consumo o en el retraso del primer contacto con la droga en aquellos que nunca serán consumidores problemáticos.

Ahora bien, muchos dirán que si los programas se aplican de forma más prolongada en todos los niveles de edad, la eficacia sería mucho mayor en todo tipo de jóvenes, consumidores o no. También se argumenta que si estos programas tienen un impacto, aunque sea pequeño, en algunos potenciales consumidores futuros de drogas, estaría más que justificada su implementación. Además, el Estado no puede evadir su responsabilidad de afrontar el problema de la droga.

En cuanto al primer argumento, hay que recordar lo que ya dijimos al principio, y es que cuando los chicos se hacen mayores se vuelven más escépticos a los mensajes que les dicen que todas las drogas son igual de perjudiciales y para cualquier nivel de consumo. Los jóvenes perciben que hay una diferencia considerable entre el consumo y el abuso de drogas, de forma que los mensajes que insisten en que todo consumo es peligroso y rechazable, en los que se basan casi todos los programas de prevención, les resultan poco creíbles, de forma que empiezan a valorar más negativamente los programas preventivos que en la preadolescencia valoraban más positivamente (Brown y Caston, 1995). En cuanto al segundo argumento, no está claro por qué está justificado que el Estado deba promover los programas de prevención de drogas aunque sólo se beneficie una minoría de jóvenes. Si nos atenemos al cannabis, que es el centro de interés de este artículo, no parece razonable que "salvar" a una cantidad determinada de jóvenes del consumo de esta droga compense el dispendio económico que supone su puesta en marcha. Y esto es así porque no parece que el consumo de cannabis represente, precisamente, un grave problema de salud pública.

Así, en nuestra muestra hemos constatado que, en general, los efectos percibidos a largo plazo de los consumidores frecuentes de cannabis (como mínimo un año de consumo) son en mayor medida positivos que negativos. En particular, la mayoría de los consumidores informa de un mayor bienestar emocional general $(61 \%)$ como consecuencia del consumo de cannabis. También, un 54\% informan de una mejora de sus relaciones personales y un $48 \%$ perciben un efecto positivo del cannabis en el nivel de comunicación, relaciones con el sexo opuesto, seguridad en sí mismo y disfrute de las cosas de la vida. Un $35 \%$ encuentra que la droga le ha ayudado a superar los problemas, mien- 
tras que un $16 \%$ ha percibido lo contrario. Por otra parte, no parece que entre los consumidores de nuestra muestra, el consumo de cannabis haya perjudicado el empleo de estrategias activas de afrontamiento del estrés. Si bien los consumidores frecuentes tienen más problemas que los no consumidores $(\mathrm{p}<.05)$ y emplean más estrategias de evitación $(\mathrm{p}<.05)$, no hay diferencias en el uso de estrategias activas para afrontar los problemas, incluso aunque más de la mitad de los consumidores $(57 \%)$ reconozcan que utilizan la droga para "desconectar" de los problemas. Al respecto hay que recordar que esta estrategia puede ser una forma útil de afrontar el estrés (Wills, 1985).

No obstante, es posible criticar estos resultados de varias formas. Primero, se basan en efectos percibidos de los consumidores, los cuales pueden no ser objetivos. Se tendría que contar, además, con otro tipo de resultados con criterios más objetivos, como análisis médicos y psiquiátricos, o con índices de búsqueda de asistencia médica por problemas agudos del consumo o derivados del consumo prolongado. Segundo, aunque pueda demostrarse en alguna medida que algunos consumidores de cannabis pueden en parte controlar su consumo y no informan de efectos negativos a largo plazo, esto no es extrapolable a los efectos muy negativos de las "drogas duras" como la cocaína y la heroína. Y tercero, la muestra es pequeña para extraer conclusiones definitivas sobre los efectos beneficiosos del cannabis.

En cuanto a la primera crítica, que faltan datos objetivos médicos y psiquiátricos, hay que decir que, al respecto, es clarificadora una magnífica revisión de las consecuencias del consumo de cannabis realizada en 1982 por el Institute of Medecine de los Estados Unidos y que, hasta la fecha, no se han obtenido resultados que alteren en una medida significativa sus conclusiones. Una lectura detenida de los resultados recogidos por el Institute of Medecine nos muestra que hay una importante diferencia entre el consumo y el exceso o abuso de cannabis. Así, mientras no se ha encontrado prácticamente ninguna consecuencia negativa del consumo de cannabis, el abuso de los consumidores "a diario" puede llevar a procesos inflamatorios crónicos en los bronquios y úvula, frecuentemente asociados con sinusitis crónica, pero que son reversibles al cesar el consumo. No hay resultados consistentes respecto a los potenciales daños sobre la función reproductiva masculina, teratogeneidad, efectos genéticos y sobre el sistema inmunitario. En cuanto a los consecuencias negativas del sistema cardiovascular, sólo se han constatado en personas con graves alteraciones de este sistema, que normalmente pertenecen a un grupo de edad distinto del de los consumidores. De especial relevancia son las consecuencias negativas sobre la conducta social y la salud mental. Así, se han descrito reacciones de pánico y fuerte ansiedad y 
descoordinación tras el consumo de dosis normales, pero estos síntomas remiten al poco tiempo (unas 4 horas) y el número de admisiones hospitalarias por este motivo es despreciable. En cuanto a fuertes reacciones disfóricas o síndrome cerebral agudo, sólo se ha constatado en grandes consumidores y con dosis muy elevadas, especialmente de hashis. La ocurrencia de "flashbacks" o de brotes psicóticos tras el consumo se ha observado en consumidores previos de LSD o en personas altamente predispuestas (esquizofrénicos) respectivamente.

Esta distinción fundamental entre las consecuencias del consumo y del abuso se pone también de manifiesto en otra serie de estudios. Así, Newcomb y Bentler (1988), en un estudio que pretendió especialmente establecer relaciones causales entre el consumo de drogas y sus consecuencias, no encontraron un impacto negativo años después del consumo de drogas (donde estaba muy representado el consumo de cannabis) en salud mental ni en relaciones sociales, pero sí unas consecuencias negativas de los grandes consumidores en aspectos particulares de la salud mental, como el aumento de la tendencia psicótica o la ideación suicida, y un mayor aislamiento social y soledad. Es curioso observar que estos autores encontraron que el consumo de alcohol, si no se consume con otras drogas ilegales, tuvo efectos muy positivos tanto en salud mental (reducción de la depresión y mejora del estado de ánimo) como en las relaciones sociales (aumento de las relaciones sociales y reducción de la soledad). Nuevamente se pone de relieve que consumir cannabis tiene unas consecuencias a largo plazo muy diferentes al hecho de abusar del cannabis.

¿Pero qué pasa con las correlaciones altas que se encuentran continuamente en la literatura científica entre la delincuencia y el cannabis, y entre el fracaso escolar, el menor interés por los estudios y la falta de interés por los proyectos futuros y el cannabis? Si bien no podemos extendernos en estas cuestiones, sí al menos daremos una sucinta respuesta aclaratoria. Así, sí es cierto que la delincuencia y las demás actitudes citadas hacia la escuela y el futuro (todo lo cual recibe el nombre de "síndrome amotivacional") se relacionan con el consumo de cannabis. Ahora bien ¿Esto dice algo de la relación causal? ¿Es la droga la que provoca estos problemas, o más bien son las actitudes previas las que conducen al consumo? Entre los numerosos estudios longitudinales de estudiantes entre 12-21 años, revisados por Kandel (1978b, c , 1980), se muestra que una serie de factores tradicionalmente relacionados con el consumo de cannabis, como son bajo rendimiento académico, delitos, baja autoestima, humor depresivo, rebeldía y otras características de personalidad, en realidad preceden al consumo de cannabis. Un estudio (Jessor y Jessor, 1977) demostró, además, que el consumo de marihuana podía agravar o exagerar cier- 
tas conductas, como la delincuencia o el bajo interés académico. No obstante, el aumento de la conducta desviada y el bajo interés escolar cambiaron más bruscamente el año que precedió al consumo.

Como conclusión de esta revisión de los efectos a largo plazo del consumo y de los antecedentes del mismo, podemos concluir que muchos males que se asocian con el consumo de cannabis (delincuencia, fracaso escolar, etc.) son parte de un sistema de valores que en realidad preceden al consumo y no son su consecuencia. En este grupo de jóvenes, el consumo de drogas ilegales es un aspecto más de su conducta desviada, y una forma más de mostrar su inconformismo con las normas sociales establecidas por los adultos. Por tanto, no es extraño que los programas de prevención del consumo de drogas no sean muy efectivos, ya que focalizar sus esfuerzos en combatir el consumo de droga supone atacar un comportamiento que forma parte de un sistema más amplio y complejo de valores y actos sociales, que además están relacionados con variables de personalidad estables. Recordemos que en nuestra muestra, el Psicoticismo y el Inconformismo están muy relacionados $(\mathrm{r}=.43, \mathrm{p}<.001)$. Por otra parte, ha quedado claro que el consumo de cannabis no tiene especiales consecuencias a largo plazo, incluso puede resultar beneficioso para una buena parte de los consumidores, mientras el abuso y exceso puede ser perjudicial a corto y largo plazo. Ya que, de alguna forma, los jóvenes reconocen esta realidad (Brown y Caston, 1995), la insistencia de los programas de prevención en los mensajes anti-droga de cualquier nivel de consumo representan más una apreciación moral sobre el consumo de drogas por parte de quienes los llevan adelante y de los responsables políticos que una actitud fundamentada científicamente.

Por todo lo dicho, y con especial referencia al cannabis, la necesidad y eficacia de los programas de prevención del consumo de drogas y las consecuencias negativas para la salud son más un mito que una realidad. Ni se ha comprobado suficientemente que los programas preventivos sean eficaces ni que el cannabis represente un problema de salud pública. El reconocimiento de que las consecuencias a largo plazo pueden ser negativas si se abusa del cannabis pero no si se consume, y la percepción ajustada a la realidad que los jóvenes tienen al respecto, nos tiene que hacer reflexionar sobre la conveniencia de afrontar el "problema de la droga" desde la información veraz de las consecuencias negativas del abuso de drogas y, en particular, del cannabis (los llamados programas de "reducción del daño") mientras se reconoce su inocuidad e incluso el beneficio de un consumo ocasional o moderado. 
Esta conclusión que acabamos de esbozar nos lleva a la propuesta de una nueva política de drogas, con programas de información de las consecuencias del mal uso y el abuso de drogas y con la no injerencia de los organismos públicos en el consumo y distribución a escala reducida del cannabis. Tal vez el modelo holandés de no intervención respecto al consumo y la permisividad y control parcial de su distribución, sea un modelo muy apropiado que se ajusta a los resultados extraídos de este estudio. La despenalización de su consumo y la permisividad de la venta a pequeña escala ha permitido alejar a esta droga de la criminalidad, pero sin aumentar desproporcionadamente su consumo. Así, si comparamos la prevalencia de vida del cannabis en Amsterdam (1987) con el de Nueva York (1988) en jóvenes de 18 años y mayores, observamos que es menor en Amsterdam $(23,7 \%)$ que en Nueva York (29\%) a pesar de la fuerte actuación policial sobre el consumo y venta de cannabis en esta última ciudad. También hay diferencia si consideramos el consumo reciente, donde encontramos un $11 \%$ en Nueva York frente a un 9,4\% en Amsterdam (Cohen, 1989). Por otra parte, no se ha constatado que la despenalización de su consumo en Amsterdam a principios de los años 70, haya conducido en el presente a un "explosión" de consumo ni a un aumento considerable del consumo de otras drogas (Cohen, 1995 ; Cohen y Sas, 1996). Al comparar muestras de 1987, 1990 y 1994 de consumidores de cannabis en Amsterdam, estos autores encontraron una estabilidad en porcentajes de prevalencia de vida por grupos de edad, alrededor de un $3 \%$ entre $12-15$ años y de un $25 \%$ entre 16 y 19 años. Sólo se ha producido un aumento del $40 \%$ en 1987 al $50 \%$ en 1994 del grupo de edad 19-24 años, pero el patrón de consumo de estos jóvenes permanece estable, es decir, aunque ha aumentado la proporción de consumidores, lo que aumenta es el consumo ocasional y moderado, no el continuado. A partir de los 24 años decrece progresivamente el interés por el cannabis. El consumo continuado (consumo en el mes anterior a la encuesta) representa entre $20-25 \%$ del total de los que han experimentado con la hierba, pero sólo el $4 \%$ son fumadores "fuertes" (más de 20 veces al mes). Curiosamente, la edad promedio del primer consumo es 20 años (no se ha reducido la edad del primer contacto con el cannabis) y la del abandono del consumo es 26 años. Además, la cantidad total de jóvenes que han solicitado tratamiento por problemas relacionados con el consumo de cannabis aumentó de 27 en 1988 a 54 en 1994. Si calculamos unos 60.000 consumidores de cannabis en Amsterdam, no se puede hablar precisamente de un problema de salud pública (Cohen, 1995). Así, parece que una política más permisiva con el consumo de cannabis y su distribución a pequeña escala (lo cual reduce su adulteración ya que los pequeños vendedores tienen que mantener un buen producto para ser "competitivos") junto con 
campañas de información de los efectos negativos del mal uso y consejos para evitar estos inconvenientes, merece una mayor atención y reconocimiento en nuestro país.

Ahora bien, si esto puede ser cierto con el cannabis, ¿qué pasa con las demás drogas? Esto nos lleva a responder a la segunda pregunta que nos hacíamos más arriba, referente a que los resultados obtenidos, de este y otros estudios y revisiones sobre el cannabis, pueden no ser aplicables a otras "drogas duras". En principio, hay que aclarar que para la mayor parte de las autoridades políticas y sanitarias que promueven las campañas de prevención contra la droga, no hay distinción entre drogas duras y drogas blandas. Así, el cannabis sería una droga igual de peligrosa que la cocaína o la heroína, sino por sus efectos, sí porque el consumo de cannabis conduce claramente al consumo de las otras drogas. Hay que recordar que ni en este estudio ni en los que se han realizado para valorar la hipótesis de la escala de las drogas desde el cannabis, se ha encontrado la confirmación de tal hipótesis. Además, en este estudio hemos constatado que los consumidores frecuentes de cannabis informan de un nivel importante de control del consumo y de beneficios a largo plazo derivados del mismo, lo cual parece inverosímil para las demás drogas. No obstante, hay una creciente evidencia de que entre los consumidores de cocaína que no están implicados en actividades delictivas ni están en centros de tratamiento, el control del consumo y la ausencia de problemas a largo plazo derivados del consumo, parece ser la norma más que la excepción (Cohen y Sas, 1992 ; Cohen y Sas, 1994 ; Erickson y Weber, 1994, Reinarman, Murphy y Waldorf, 1994 ). Estos mismos autores concluyen que estos resultados apuntan a la necesidad de estudiar políticas de drogas alternativas a la política prohibicionista extendida en todo el mundo. No obstante, más investigación es necesaria sobre esta cuestión.

La tercera crítica a las conclusiones extraídas de este estudio, a la que hicimos referencia más arriba, se refería a que no es posible sacar conclusiones importantes de una muestra relativamente reducida. Ahora bien, este estudio cumple la función de explorar desde otra óptica distinta de la tradicional el estudio del "problema de la droga", obteniendo resultados congruentes, en buena parte, con la evidencia experimental existente y que aquí ha sido sucintamente expuesta. Además, abre nuevas estrategias de investigación que ya están dando su fruto. Así, los primeros resultados que están siendo evaluados en este momento de un estudio en marcha en un Instituto de BUP y COU, en el que se ha administrado la encuesta que aquí se propone, están replicando los resultados más sobresalientes que se han obtenido aquí. Así, entre los consumidores actuales de cannabis $(\mathrm{N}=46)$ de un total 
de 360 alumnos que respondieron a la encuesta, se está encontrando que la mayoría acepta mejor los mensajes permisivos sobre el consumo que los punitivos, siendo que la mayoría de los no consumidores, prefiere los punitivos. Además, los consumidores informan, en general, de consecuencias favorables del consumo prolongado de cannabis más que de consecuencias negativas. También se han replicado las diferencia significativas en Psicoticismo e Inconformismo entre los consumidores y no consumidores, al igual que el poder predictivo de estas variables sobre el consumo, abuso y preferencia de mensajes permisivos. Los datos están todavía analizándose y que serán presentados en un futuro, pero, como podemos ver, los resultados están replicando lo aquí encontrado.

\section{BIBLIOGRAFÍA}

ARGYLE, M. y LU, L. (1990): The happiness of extroverts. Personality and Individual Differences, 11, 1011-1017.

BANDERT-DROWNS, R.L. (1988): The effects of school-based substance abuse education. A meta-analysis. Journal of Drug Education, 18, 243264.

BECKER, H.K., AGOPIAN, M.W. y YEH, S. (1992). Impact evaluation of Drug Abuse Resistance Education (DARE). Journal of Drug Education, 22, 283-291.

BIGLAN, A., WEISSMAN, W. y SEVERSON, H. (1985): Coping with social influences to smoke. En S. Shiffman and T.A. Wills (Eds.), Coping and substance abuse. New York and London: Academic Press. pp 95-116. BROWN, J.H. y CASTON, M.D. (1995): On becoming "at risk" through drug education. How symbolic policies and their practices affect students. Evaluation Review, 19, 451-492.

CATTARANZO, S.J. y MEARNS, J. (1990): Measuring Generalized Expectancies for Negative Mood Regulation: Initial Scale Development and Implications. Personality Assessment, 54, 546-563.

CLAYTON, R.R., CATTARELLO, A., DAY, L.E. y WALDEN, K.P. (1991): Persuasive communications and drug abuse prevention: An evaluation of the DARE program. En L. Donohew, H. Sypher y W. Bukowsky (Eds.), Persuasive communication and drug abuse prevention. Hillsdale, NJ: Lawernce Erlbaum. Pp 295-313.

CLAYTON, R.R., CATTARELLO, A. y WALDEN, K.P. (1991): Sensation seeking as a potential mediating variable for school based prevention interventions: A two year follow-up of DARE. Journal of Health Communications, 3, 229-239.

COHEN, P. (1989): Cocaine use in Amsterdam in non-deviant subcultures. Amsterdam: University of Amsterdam. 
COHEN, P. (1990): Cocaine and Cannabis: An identical policy for different drugs? En P. Cohen (Eds.), Drugs as a social construct. Amsterdam; CEDRO.

COHEN, P. (1995): Cannabis users in Amsterdam. Presentación en la Conference on Urban Softdrugs Tolereance Polivy, Jaarbeurs Conference Center, Utrecht.

COHEN, P. y SAS, A. (1996): Cannabis use, a stepping stone to other drugs? The case of Amsterdam. Amsterdam: CEDRO.

COHEN, P. y SAS, A. (1992): Loss of control over cocaine, rule or exception? Paper presented at the American Society of Criminology, New Orleans.

COHEN, P. y SAS, A. (1994): Cocaine use in Amsterdam in non deviant subcultures. Addiction Research, 2, 71-94.

DEMBO, R. (1979): Substance abuse prevention programming and research: A patnership in need of improvement. Journal of Drug Education, 9, 189208.

DEMBO, R. (1981): Critical issues and experiences in drug treatment and prevention evaluation. International Journal of Addictions, 16, 1399-1414.

DUKES, R.L., ULLMAN, J.B. y STEIN, J.A. (1996): Three-year follow-up of Drug Abuse Resistance Education (DARE). Evaluation Review, 20, 4966.

DUKES, R.L., ULLMAN, J.B.y STEIN, J.A. (1995): An evaluation of D.A.R.E (Drug Abuse Resistance Education) using a Solomon four group design with latent variables. Evaluation Review, 19, 409-435.

ENNET, S.T., ROSEMBAUM, D.P., FLEWELLING, R.L., BIELER, G.S., RINGWALT, C.L. y BAILEY, S.L. (1994): Long-term evaluation of Drug Abuse Resistance Education. Addictive Behaviors, 19, 113-125.

ENNET, S.T., TOBLER, N.S., RINGWALT, C.L. y FLEWELLING, R.L. (1994): How effective is Drug Abuse Resistance Education? A metaanalysis of project DARE outcome evaluations. American Journal of Public Health, 84, 1394-1401.

ERICKSON, PG. y WEBER, T.R. (1994): Cocaine careers, control and consequences; results from a canadian study. Addiction Research, 2, 3750.

ESCÁMEZ, J. (1990): Drogas y escuela: Una propuesta de prevención. Madrid: Dykinson.

EYSENCK, H.J. y EYSENCK, S.B.G. (1964): Eysenck Personality Inventory. London: Hodder and Stoughton, Educational.

EYSENCK, H.J. y EYSENCK, S.B.G. (1975): Manual of the Eysenck Personality Questionnaire (junior \& adult). London: Hodder and Stoughton.

FAINE, J.R. y BOHLANDER, E. (1989): DARE in Kentucky schools 198889; An evaluation of the Drug Abuse Resistance Education program. Bowling Green, KY: Western Kentucky University Social Research Laboratory. 
FISHBURNE, P.M., ABELSON, H.I. y CISIN, I. (1980): National Survey on Drug Abuse: Main Findings:1979. DHHS Publication No. (ADM) 80976. Washington, D.C.: U.S. Government Printing Office.

FROJÁN, M. y SANTACREU, J. (1994): Evaluación de programas de prevención del consumo de drogas. Adiciones, 6, 283-299.

GOODSTADT, M.S. (1978): Alcohol and drug education: Models and outcomes. Health Education Monographs, 6, 263-279.

GOODSTADT, M.S., SHEPPARD, M.A. y CHAN, G.C. (1982): Relationships between drug education and drug use. Carts and horses. Journal of Drug Issues, 431-442.

HOLAHAN, C.J. y MOOS, R.H. (1987): Personal and contextual determinants of coping strategies. Journal of Personality and Social Psychology, 52, 946-955.

INSTITUTE OF MEDECINE (1982): Marihuana and Health. Washington: National Academy Press.

JESSOR, R. y JESSOR, S.L. (1977): Problem Behavior and Psychological Development: A Longitudinal Study of Youth. New York: Academic Press.

KANDEL, D.B. (1978a): Homophily, selection, and socialization in adolescent friendship. American Journal of Sociology, 84, 427-436.

KANDEL, D.B. (1978b): Longitudinal Research on Drug Use: Empirical Findings and Methodological Issues. Washington, D.C.: Hemisphere Publishing Corp.

KANDEL, D.B. (1978c): Convergences in prospective longitudinal surveys of drug use in normal populations. En D.B Kandel (Eds.), Longitudinal Research on Drug Use: Empirical Findings and Methodological Issues. Washington, D.C.: Hemisphere Publishing Corp. Pp 3-38.

KANDEL, D.B. (1980): Drug and drinking behavior among youth. Annual Review of Sociology, 6, 235-285.

KANDEL, D.B., YAMAGUCHI, K. Y CHEN, K. (1992): Stages of progression in drug involvement from adolescence to adulthood: further evidence for the Gateway Theory. Journal of Studies on Alcohol, 447-452.

MACIÁ, D. (1986): Método conductual de prevención de la drogodependencia. Valencia: Promolibro.

MCKAY, M., DAVIS, M. y FANNING P. (1981): Thoughts and Feelings, the Art of Cognitive Stress Intervention. New Harbinger Publications.

NEWCOMB, M.D. y BENTLER, P.M. (1988): Consequences of adolescent drug use. Impact on the lives of young adults. London: Sage Publications.

PENTZ, M.A, (1985): Social competence and self-efficacy as determinants of substance abuse in adolescence. En S. Shiffman and T.A. Wills (Eds.), Coping and substance abuse. New York and London: Academic Press. pp 117-142.

REINARMAN, C., MURPHY, S. y WALDORF, D. (1994): Pharmacology is not destiny: The contingent character of cocaine abuse and addction. Addiction Research, 2, 21-36. 
ROSEMBAUM, D.P., FLEWELLING, R.P., BAILEY, S.L., RINGWALT, C.L. y WILKINSON, D.L. (1994): Cops in the classroom: A longitudinal evaluation of Drug Abuse Resistance Education (DARE). Journal of Research in Crime and Delinquency, 31, 3-31.

SMART, R.G. (1983): The Nature, Treatment and Prevention of Illicit Drug Abuse. Toronto: Adiction Research Foundation.

TIMMER, S.G., VEROFF, J. y COLTEN, E.E. (1985): Life strees, helplessness, and the use of alcohol and drugs to cope: an analysis of National Survey Data. En S. Shiffman and T.A. Wills (Eds.), Coping and substance abuse. New York and London: Academic Press. pp 171-198.

WATSON, D., CLARK, L.A. y TELLEGEN, A. (1988): Development and validation of brief measures of positive and negative affect: the PANAS Scales. Journal of Personality and Social Psychology, 54, 1063-1070.

WILLS, T.A. (1985): Stress, coping, and tobacco and alcohol use in Early adolescence. En S. Shiffman and T.A. Wills (Eds.), Coping and substance abuse. New York and London: Academic Press. pp 67-94.

WYSONG, E., ANISKIEWICZ, R. y WRIGHT, D. (1994): Truth and DARE: Tracking drug education to graduation and as symbol politics. Social Problems, 41, 448-472. 